\title{
Macroscopic yielding of oriented polymers
}

\author{
ROBERT M. CADDELL, ALAN R. WOODLIFF \\ Department of Mechanical Engineering. The University of Michigan, Ann Arbor, \\ Michigan, USA
}

Uniaxial and biaxial stress states were employed to compare the measured macroscopic yield behaviour of oriented polycarbonate, polyethylene, and polypropylene with a proposed anisotropic and pressure-dependent yield criterion. A good correlation between theory and experiment was found for each material.

\section{Introduction}

Since the purpose of this paper is to discuss certain aspects about the macroscopic yield behaviour of oriented or anisotropic polymers, no reference will be made to the many studies involving unoriented polymers.

In comparison with the amount of research in which unoriented polymers have been used, there are relatively few investigations that have reported findings on the macroscopic yielding of oriented polymers. Most of these [1-4] have followed the suggestions of Hill [5] wherein the variations in tensile and compressive yield strengths of oriented sheet materials have provided the means for comparing analytical predictions with experimental results. As discussed in a subsequent article [6], these earlier studies were questioned on fundamental grounds.

The yield criterion for anisotropic and pressure dependent solids proposed elsewhere [6] was investigated in a limited study [7] in which first and fourth quadrant points were determined so as to compare a theoretical yield locus with experimental results. Because the correlation was quite reasonable, the validity of this proposed yield criterion seemed quite promising and prompted a more extensive investigation. The findings of this latter study provide the basis of this present paper.

\subsection{Nomeclature}

$$
\begin{aligned}
\sigma & =\text { true normal stress. } \\
\tau & =\text { shear stress. } \\
\epsilon & =\text { true normal strain. } \\
L & =\text { load. }
\end{aligned}
$$

$A_{0}=$ original area.

$l=$ instantaneous length.

$l_{0}=$ original length .

$C=$ absolute value of compressive yield stress at atmospheric pressure and room temperature.

$T=$ absolute value of tensile yield stress at atmospheric pressure and room temperature.

$H, F, G, N, L, M=$ anisotropic parameters.

$K_{1,2,3}=$ pressure modifying parameters.

$R_{1}=$ axial stress normalized with respect to $T_{1}$.

$R_{2}=$ circumferential (hoop) stress normalized with respect to $T_{1}$.

Subscripts $x, y, z=$ refer to a general orthoganal co-ordinate system.

Subscripts 1,2,3= principal directions.

\section{Analytical development}

A full development of the yield criterion proposed for use with pressure dependent, anisotropic solids is presented elsewhere [6] and need not be repeated here. It is, hopefully, sufficient to indicate that the modification proposed by Hill [5], which altered the standard von Mises criterion to account for anisotropic effects, has been further modified to account for pressure dependency of yielding.

In its most general form, this suggested criterion is expressed as

@ 1977 Chapman and Hall Ltd. Printed in Great Britain. 


$$
\begin{aligned}
H\left(\sigma_{x}-\right. & \left.\sigma_{y}\right)^{2}+F\left(\sigma_{y}-\sigma_{z}\right)^{2}+G\left(\sigma_{z}-\sigma_{x}\right)^{2} \\
& +2 N \tau_{x y}^{2}+2 L \tau_{y z}^{2}+2 M \tau_{z x}^{2}+K_{x} \sigma_{x} \\
& +K_{y} \sigma_{y}+K_{z} \sigma_{z}=1 .
\end{aligned}
$$

The parameters $H, F, G, N, L$ and $M$ characterize, in their usual sense [5], the state of anisotropy while those denoted as $K_{x}, K_{y}$ and $K_{z}$ account for pressure dependency. All of these parameters are defined as functions of the absolute values of the compressive and tensile yield strengths measured in the three reference directions at atmospheric pressure and room temperature.

Since yield locus studies involve biaxial states of stress application, and using the usual notation involving principal directions, Equation 1 reduces to

$$
\begin{aligned}
(H+G) \sigma_{1}^{2}+(H+F) \sigma_{2}^{2}-2 H \sigma_{1} \sigma_{2}+K_{1} \sigma_{1} \\
+K_{2} \sigma_{2}=1 .
\end{aligned}
$$

In developments that follow it is assumed that the condition of anistropy is symmetric about the axis of orientation. This has been found to be quite an acceptable assumption in our work and is discussed in some detail at a later point. As the work reported in this paper employed thin-walled tubes that were subjected to the simultaneous loading of internal pressure and axial forces in order to develop biaxial stress states, the assumption of aixal symmetry meant that the radial and hoop yield stresses were equivalent. Considering the axial direction (this corresponds to the direction in which orientation was induced) as the 1-direction, and the 2-direction as the hoop or circumferential

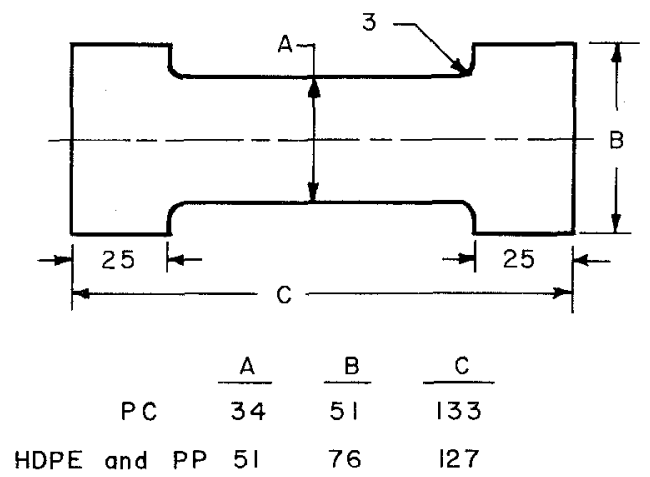

Figure 1 Dimensions (mm) of specimens prior to orientation. direction, the basic yield strengths measured at atmospheric pressure are expressed as follows $T_{1}$ and $C_{1}$ (usually not equal in magnitude) refer to axial direction, $T_{2}=T_{3}$ and $C_{2}=C_{3}\left(T_{2} \neq C_{2}\right)$ refer to hoop direction.

In regard to equation 2 , the various parameters are defined as:

$$
\begin{gathered}
H+G=\frac{1}{T_{1}\left|C_{1}\right|}, \\
F+H=G+F=\frac{1}{T_{2}\left|C_{2}\right|}=\frac{1}{T_{3}\left|C_{3}\right|} \\
K_{1}=\frac{\left|C_{1}\right|-T_{1}}{\left|C_{1}\right| T_{1}} \\
K_{2}=K_{3}=\frac{\left|C_{2}\right|-T_{2}}{\left|C_{2}\right| T_{2}}=\frac{\left|C_{3}\right|-T_{3}}{\left|C_{3}\right| T_{3}}
\end{gathered}
$$

As seen in Equation 3, $H=G$ as a consequence of rotational symmetry about the axis of orientation.

\section{Test materials and method of producing an oriented structure}

The three polymers used were polycarbonate (PC), high density polyethylene (HDPE) and polypropylene (PP). They were obtained from the Westlake Plastics Company in the form of solid cylindrical rods whose original diameters were $\sim 51$ (PC) and $64 \mathrm{~mm}$ (PP and HDPE). From every individual bar used, a section was cut off for use with "unoriented characterization studies" to be discussed later. The remainder of each bar was then employed to produce oriented material by first machining oversized tensile specimens to the dimensions shown in Fig. 1. Each specimen was loaded in uniaxial tension until a stable neck formed and propagated to a length of about $64 \mathrm{~mm}$. Subsequent machining of a thin-walled tube followed such that the tube test section of about $51 \mathrm{~mm}$ lay completely within the highly oriented (necked) region. Fig. 2 typifies this sequence of operations.

It is of possible interest to mention procedural details that were found necessary to avoid problems when producing an adequate length of oriented material by the technique used in this work. As mentioned elsewhere [7], PC has a susceptibility to surface cracking unless care is taken in specimen preparation. This may be accomplished with the use of a large radius turning tool followed by

\footnotetext{
*In Equations 3 and 4 the absolute value signs are included to aovid possible confusion; it is the magnitude of the compressive values that are important. For simplicity, these absolute value signs will not be used through out the remainder of the paper.
} 


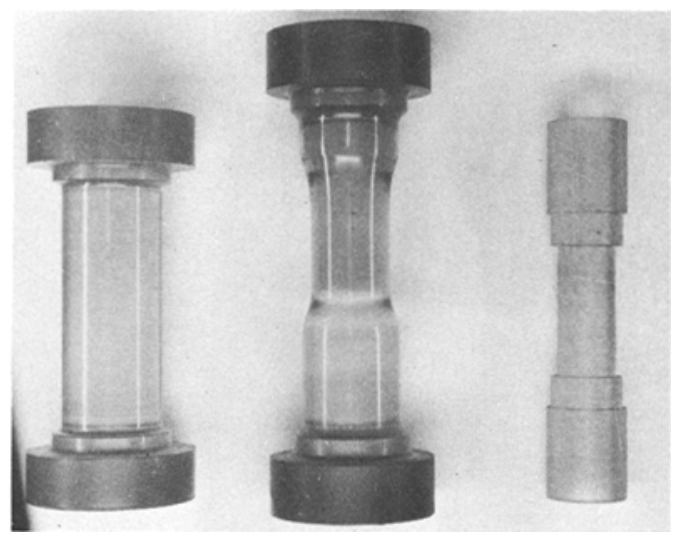

Figure 2 Initial specimen unoriented, specimen after orientation caused by neck propagation, and typical tubular specimen after being machined from oriented specimen.

polishing with a buffing compound so as to eliminate all "feed" marks from the initial turning operation. In addition, a low crosshead-loading speed (we used about $22 \mu \mathrm{m} \mathrm{sec}^{-1}$ ) must be employed to prevent premature fracture as the neck begins to form. For all specimens so produced, the maximum true strain of the necked region was on the order of 0.53 which implied that the degree of orientation was consistent among all test specimens.

HDPE shows almost no susceptibility to surface cracking and no polishing is necessary after the specimen has been turned to size. Higher crosshead speeds may be used $\left(110 \mu \mathrm{m} \mathrm{sec}^{-1}\right)$ during the orientation process, and all necked specimens displayed a true strain very close to 1.86 . The only precaution to be observed relates to using too small an initial test section which would require the neck to propagate close to the original shoulder of the specimen. This can lead to the type of cracking shown in Fig. 3. By using the specimen dimensions in Fig. 1, this result was avoided.

The behaviour of PP was similar to HDPE in most respects. A crosshead speed of $110 \mu \mathrm{m} \mathrm{sec}^{-1}$ was used and in all specimens displayed a true strain of about 1.83 in the necked region.

\section{Characterization of test materials}

Certain documentation of the three materials was provided by the supplier. The PC was made from Lexan Resin 101 and marketed under the name "Zelux". It had a specific gravity of 1.20 , yield strength of $62 \mathrm{MPa}$, tensile and compressive modu-

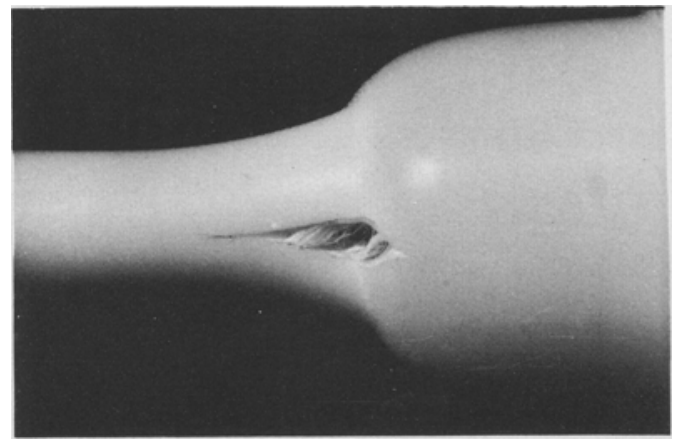

Figure 3 Oriented HDPE specimen which cracked as a result of the neck propagating too near the shoulder.

lus of $2.38 \mathrm{GPa}$ and Rockwell $\mathrm{M}$ hardness of 70 . The HDPE was made from Phillips "Marlex" 5003 and marketed by the supplier under the name "Ultra Ethylux". This is a linear polyethylene whose specific gravity was 0.95 and melt index 0.3 . The weight average molecular weight was 50000 and test bars were solidified by air cooling. The PP was made from Moplen D004W and marketed under the name "Propylux". This is a homopolymer having a density of $0.905 \mathrm{Mg} \mathrm{m}^{-3}$ at $23^{\circ} \mathrm{C}$, melting point of $168^{\circ} \mathrm{C}$, yield strength of 33.8 MPa and Rockwell $\mathrm{R}$ hardness of 92 .

To determine the extent of uniformity of structure of the cross-section (and thus, indirectly, the extent of rotational symmetry about the rod axis), a number of "microscopic" and "macroscopic" tests were made. With the unoriented material (i.e. the original test bars), specimens were selected from regions near the outer diameter and centre of each bar at several longitudinal locations. For the oriented material, surface and centre specimens were selected from the highly strained neck region. The results of these tests should indicate if any meaningful variations existed across a diametral section either because of the original processing or because of non-uniformities that might arise from the formation of a stable neck.

\subsection{Microscopic studies}

Three types of measurements were used and are listed as follows: (1) Wide-angle X-ray diffraction (WAXD) patterns were obtained with a Statton Flat Plate Unit. (2) A Perkin-Elmer differential scanning calorimeter (DSC) served for calorimetric measurements; (3) Density measurements were, in all but one case, determined with a density gradient column and glass beads of standard densities. 
Because the oriented PP sample floated on the top of the column, a carefully machined right circular cylinder was accurately measured and weighed on a chemical balance to obtain a reasonable measure of density.

Fig. 4 typifies the WAXD patterns for oriented PC at either surface or centre locations. Variations per structural condition as a function of radial location were found to be minimal. Fig. 5 shows typical results for oriented HDPE, while Fig. 6 typifies the results using oriented PP. None of the unoriented materials displayed any arcing regardless of specimen location, thereby indicating that variations in molecular orientation due to original processing were insignificant.

Table I lists the results from the DSC and density experiments. It can be seen that for all three materials and for each structural condition, the variation in any measurement as a function of radial location is truly minimal.

\subsection{Macroscopic studies}

From every original (unoriented) bar, a number of uniaxial tensile and compressive tests were conducted using specimens produced from at least

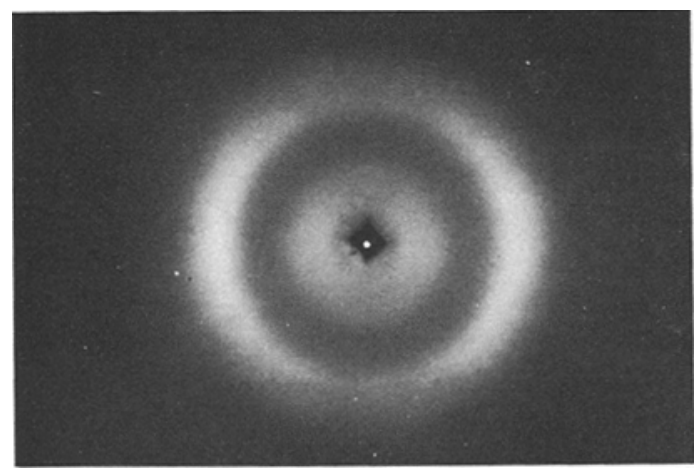

Figure 4 WAXD pattern for oriented PC.

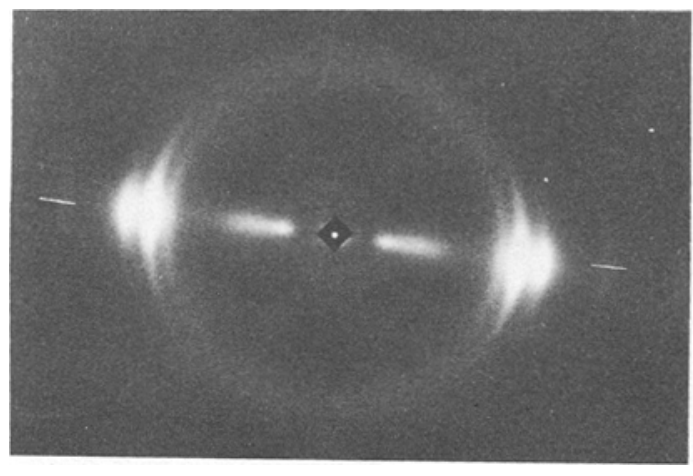

Figure 5 WAXD pattern for oriented HDPE.

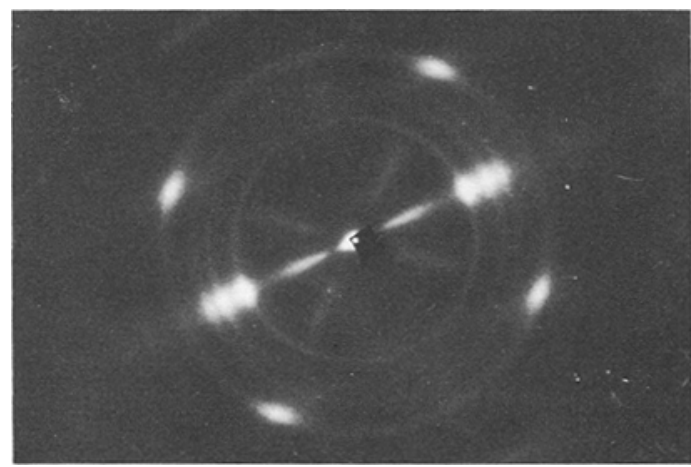

Figure 6 WAXD pattern for oriented PP.

three radial locations across the cross-section. Regardless of the test material, any variations per location were found to be minimal for either tensile or compressive loading. Raw data were converted into true stress-true strain values and Fig. 7 typifies the degree of consistency observed. Since the microscopic results implied rotational symmetry of structure for both the unoriented and oriented conditions, and macroscopic results agreed with those findings for the unoriented con-

TABLE I Density and DSC measurements at surface (S) and centre (C) locations

\begin{tabular}{|c|c|c|c|c|c|c|c|c|}
\hline \multirow[t]{3}{*}{ Material } & \multicolumn{4}{|c|}{ Density $\left(\mathrm{Mg} \mathrm{m}^{-3}\right)$} & \multicolumn{4}{|c|}{$T_{\mathrm{m}}$ or $T_{\mathrm{g}}\left({ }^{\circ} \mathrm{C}\right)^{\dagger}$} \\
\hline & \multicolumn{2}{|c|}{ Unoriented } & \multicolumn{2}{|c|}{ Oriented } & \multicolumn{2}{|c|}{ Unoriented } & \multicolumn{2}{|c|}{ Oriented } \\
\hline & $\mathrm{S}$ & $\mathrm{C}$ & $\mathrm{S}$ & $\mathrm{C}$ & $\mathrm{S}$ & C & $S$ & C \\
\hline PC & 1.196 & 1.196 & 1.197 & 1.197 & 155 & 152 & 155 & 157 \\
\hline HDPE & 0.954 & 0.954 & 0.942 & 0.942 & 131 & 133 & 134 & 131 \\
\hline PP & 0.909 & 0.909 & * & 0.734 & 161 & 161 & 162 & 161 \\
\hline
\end{tabular}

*A transverse specimen, which included both surface and centre material, was measured then weighed on a chemical balance. Its density was $0.800 \mathrm{Mg} \mathrm{m}^{-3}$ indicating that a probable void formation was not uniform across the necked region. In [4] such void formation was also noted.

†For HDPE and PP, the values refer to melting temperature, $T_{\mathrm{m}}$.

For $\mathrm{PC}$, the values refer to the glass transisiton temperature, $T_{\mathrm{g}}$. 
dition, the assumption of such symmetry for the macroscopic properties of interest in regard to the oriented structure appears to be quite reasonable.

\section{Experimental test procedures}

To evaluate the parameters in Equations 3 and 4 , measurements of $T_{1}, T_{2}, C_{1}$ and $C_{2}$ were made from the oriented material.

\subsection{Tension tests}

The magnitude of $T_{1}$ was found by using unpressurized tubes that were loaded on an Instron machine whose crosshead speed was $8.33 \mu \mathrm{m} \mathrm{sec}^{-1}$. A standard extensometer was used to drive the chart recorder and the load-extension data were converted to give true stress-true strain plots.

Because of size restrictions, tension tests could not be performed on material transverse to the orientation direction. Instead, the value of $T_{2}$ was obtained using the open-ended tube method described by Radhava [8]. For all practical purposes, this approach provides only a hoop stress to be induced in the wall section as internal fluid

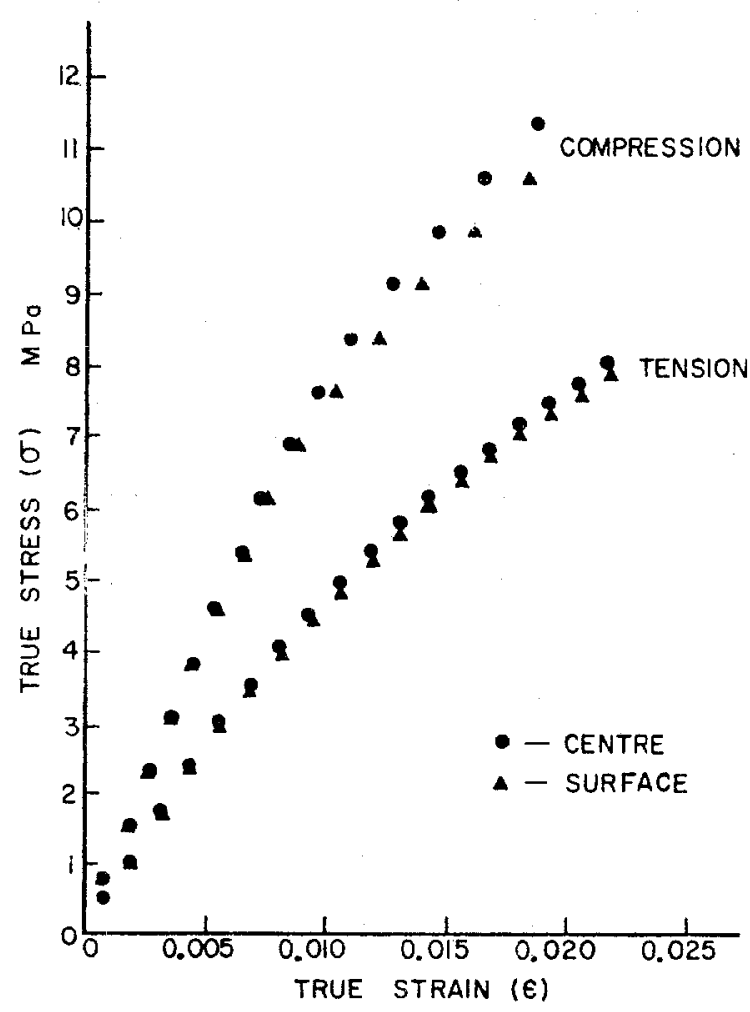

Figure 7 Uniaxial tensile and compressive true stresstrue strain plot for unoriented HDPE surface (S) and centre $(C)$ specimens. pressure is increased. An extensometer, with appropriate modifications, was adapted to measure changes in diameter concurrently with pressure in crease. This information was reduced to provide a plot of true stress--true strain, two such tests being performed.

\subsection{Compression tests}

The magnitude of $C_{1}$ was found using solid right circular cylinders machined from material in the vicinity of the region from which the wall of a tube would normally be produced. These specimens were subjected to direct compression at the same crosshead speed used for the tensile tests. Possible frictional effects at the interface were checked by running tests both with and without a lubricant, and the results were so similar that no concern was necessary. The raw load data were corrected to account for load cell deflection and other elastic deflections of the overall system. These results were then converted to give true stress-true strain plots. As a comparative check, a thin-walled tube was subjected to direct compression without the use of internal pressure but buckling problems negated this type of test.

To obtain the magnitude of $C_{2}$, solid right circular cylinders were machined from material in the necked region transverse to the orientation direction. The test procedure duplicated that used to obtain $C_{1}$. Many of the details of specimen preparation are noted elsewhere [7] and are not repeated here.

\subsection{Thin-wall tube tests}

Tubes as shown in Fig. 2 were machined to produce the necessary test section. The length of the test section was about $51 \mathrm{~mm}$ regardless of material but because the necked diameters were not the same for all materials, the outer diameter and wall thicknesses varied. For PC, the tube diameter was about $23 \mathrm{~mm}$, while the wall thickness was about $1 \mathrm{~mm}$. Tubes of HDPE and PP were machined to produce a diameter of about $17 \mathrm{~mm}$ and wall thickness of about $0.76 \mathrm{~mm}$.

During a typical test, the tube was subjected to a combination of internal pressure and axial loading in a manner that produced a reasonably constant stress ratio of $\sigma_{1}$ to $\sigma_{2}$ during deformation. Length changes in the test section were sensed by a standard $25 \mathrm{~mm}$ extensometer (either for lengthening or shortening) while diametral changes were sensed in the manner described in Section 
5.1. These electrical outputs were fed into an $X-Y-Y$ recorder which provided a time base. By correlating combinations of axial load, pressure, length change and diameter change, the necessary data for plotting true stress--true strain curves were obtained.

\section{Experimental results}

\subsection{Uniaxial tension and compression}

As found in earlier work [7], volume changes during the deformation of polymers are finite, but for the purpose of the studies being discussed, it is quite reasonable to employ the concept of volume constancy to determine values of true stress and true strain. These are found from

$$
\sigma=\frac{L l}{A_{0} l_{0}}, \text { and } \epsilon=\ln \left(l / l_{0}\right)
$$

where $\sigma$ and $\epsilon$ are the stress and strain respectively (whether tensile or compressive), $L$ and $l$ the instantaneous load and length respectively, and $A_{0}$ and $l_{0}$ refer to the initial cross-sectional area and extensometer length of the unloaded specimen. The method used to define the "yield strength" involves the use of $0.3 \%$ offset. All individual and "average" values are listed in Table II. Because buckling of the unpressurized tubes was noted in all direct compression tests, the yield strength values obtained were suspect and are not included in the "average" value of $C_{1}$.

\subsection{Thin-wall tube tests}

As discussed elsewhere [7-9], the pressure, axial load, length and diameter changes from each test were converted into values of $\sigma_{1}$ and $\sigma_{2}$ which were plotted against an effective strain function associated with the von Mises yield criterion. A typical plot is shown in Fig. 8 where the values of $\sigma_{1}$ and $\sigma_{2}$ connected with "yielding" are also found using a $0.3 \%$ offset. Definite support for this approach is found since the ratio of $\sigma_{1}$ to $\sigma_{2}$ which defines "yielding" via a plot such as Fig. 8 always agreed closely with the applied stress ratio used in a given test.

\section{Comparison af analytical predictions with experimental results}

A normalized form of Equation 2 is found to be useful when plotting yield loci. One approach is as follows:

$$
R_{1}=\sigma_{1} / T_{1}, \text { and } R_{2}=\sigma_{2} / T_{1} .
$$

Then, Equation 2 can be rewritten as

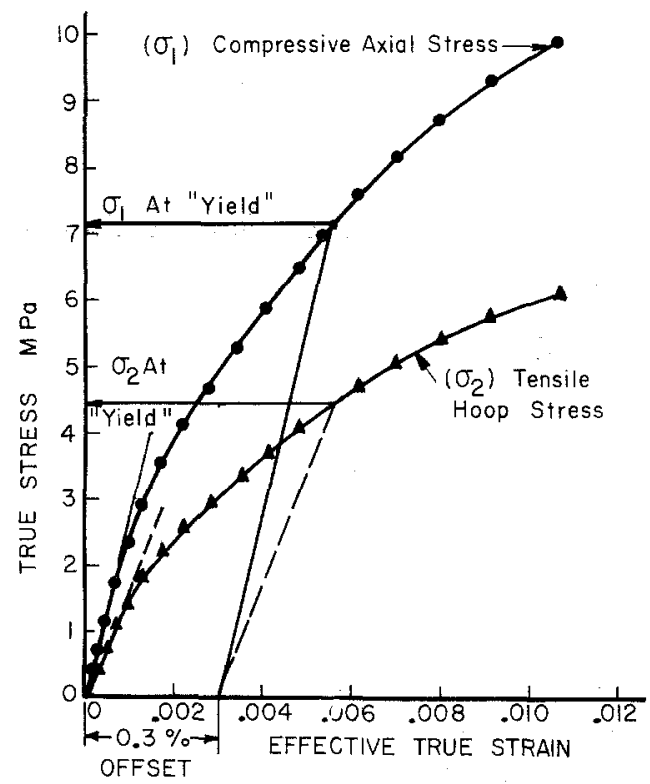

Figure 8 Biaxial stresses $\left(\sigma_{1}\right.$ and $\left.\sigma_{2}\right)$-effective true strain plot for a HDPE tube at a stress ratio $\left(\sigma_{1} / \sigma_{2}\right)$ of -1.61 at yielding.

TABLE II Yield stresses (MPa) for uniaxial tension and compression

\begin{tabular}{lllllll}
\hline Type of Test & PC & & HDPE & & PP \\
\cline { 2 - 6 } & Individual & Average & Individual & Average & Individual & Average \\
\hline $\begin{array}{l}\text { Uniaxial tension } \\
\text { with unpressurized } \\
\text { tube }\end{array}$ & $64.1,66.2$ & $T_{1}=65.2$ & $17.8,17.7$ & $T_{1}=17.7$ & $37.8,30.1$ & $T_{1}=34.0$ \\
$\begin{array}{l}\text { Hoop tension with } \\
\text { "open ended"tube }\end{array}$ & $33.4,36.9$ & $T_{2}=T_{3}=35.2$ & $9.60,10.6$ & $T_{2}=T_{3}=10.1$ & $14.8,14.1$ & $T_{2}=T_{3}=14.4$ \\
$\begin{array}{l}\text { Direct compression } \\
\text { in oriented direction }\end{array}$ & $42.0,43.4 C_{1}=42.7$ & $12.6,13.7,16.2$ & $C_{1}=14.2$ & $26.2,25.6$ & $C_{1}=25.9$ \\
$\begin{array}{l}\text { Direct compression } \\
\text { in transverse direction }\end{array}$ & $44.8,45.5$ & $C_{2}=C_{3}=45.2$ & $13.5,14.3,14.9,17.2 C_{2}=C_{3}=15.0$ & $9.65,8.76$ & $C_{2}=C_{3}=9.20$ \\
\hline
\end{tabular}




$$
\begin{gathered}
(H+G) R_{1}^{2}+(H+F) R_{2}^{2}-2 H R_{1} R_{2}+\frac{K_{1} R_{1}}{T_{1}} \\
+\frac{K_{2} R_{2}}{T_{1}}=\frac{1}{T_{1}^{2}}
\end{gathered}
$$

For the case where $C_{2}=C_{3}$ and $T_{2}=T_{3}, 2 H=$ $H+G$, so Equation 6 can be rewritten as

$$
\begin{gathered}
R_{1}^{2}+\frac{H+F}{H+G} R_{2}^{2}-R_{1} R_{2}+\frac{K_{1}}{T_{1}(H+G)} R_{1} \\
+\frac{K_{2}}{T_{1}(H+G)} R_{2}=\frac{1}{(H+G) T_{1}^{2}} .
\end{gathered}
$$

Defining new parameters as:

and

$$
\begin{gathered}
X=\frac{H+F}{H+G}=\frac{C_{1} T_{1}}{C_{2} T_{2}}, \\
Y=\frac{K_{2}}{T_{1}(H+G)}=\frac{C_{1}}{C_{2}}\left(\frac{C_{2}}{T_{2}}-1\right),
\end{gathered}
$$

$$
Z=\frac{1}{(H+G)} T_{1}^{2}=\frac{C_{1}}{T_{1}}
$$

Equation 7 becomes:

$$
R_{1}^{2}+X R_{2}^{2}-R_{1} R_{2}+(Z-1) R_{1}+Y R_{2}=Z
$$

The parameters $X, Y$ and $Z$ are evaluated for the test materials by using the values for $T_{1}, T_{2}, C_{1}$ and $C_{2}$ from Table II; Table III contains the results.

The experimental values of $\sigma_{1}$ and $\sigma_{2}$ were normalized with their respective values for $T_{1}$ and plotted in Fig. 9 to 12. Also shown on each figure is the appropriate plot of Equation 9 which uses the parameters from Table III.

TABLE III Yield loci Parameters for use in Equation 9.

\begin{tabular}{llrl}
\hline Material & $X$ & \multicolumn{1}{c}{$Y$} & \multicolumn{1}{l}{$Z$} \\
\hline Polycarbonate & 1.750 & .268 & .655 \\
Polyethylene & 1.659 & .457 & .802 \\
Polypropylene & 6.647 & -1.017 & .762 \\
Combined Polycarbonate $^{*}$ & 1.568 & .275 & .706 \\
\hline
\end{tabular}

${ }^{*}$ These include the results from the current study and those from [7].

\section{Discussion}

The correlation between experimental values and the theoretical loci in Fig. 9 to 12 seems most promising. Even when the results of a previous study [7] are included with those from this study

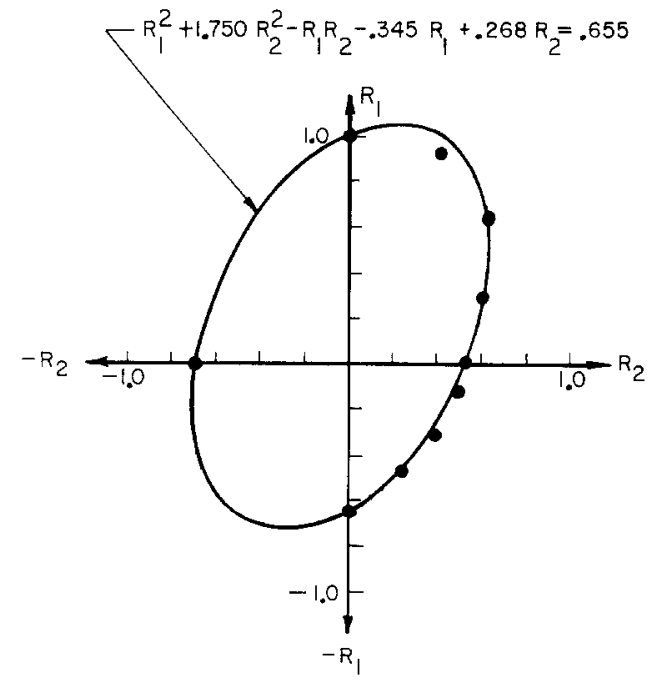

Figure 9 Comparison between theoretical yield locus based on the proposed criterion (Equation 9) and actual experimental results for oriented PC.

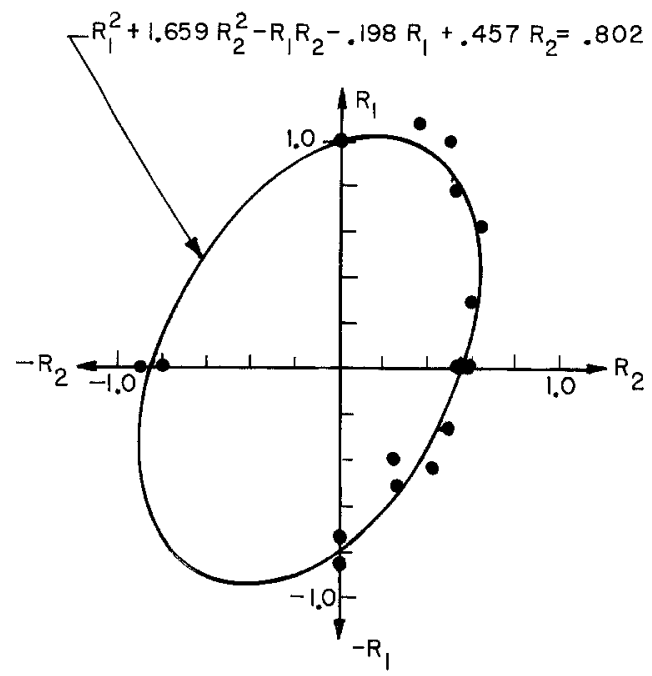

Figure 10 Comparison between theoretical yield locus based on the proposed criterion (Equation 9) and actual experimental results for oriented HDPE.

(Fig. 12), the normalized yield locus describes the macroscopic yielding very well.

Several points are worth noting about the accuracy of the values:

(1) In a direct comparison of the values from the two different studies using oriented PC, the experimental results from the present study more accurately fit the theoretical yield locus. This can be attributed to a more exact method for determining the change in diameter during the course of a test. The present study used a modified extensometer whose output was fed into an $X-Y-Y$ re- 
corder, while in the previous study [7] a micrometer was used to measure the outside diameter.

(2) The experimental points from the tests with HDPE seem to be more erratic than those where PC or PP was used. This scatter seemed to carry throughout the whole range of testing with HDPE and could be attributed to a less stringent control of processing. Also, Raghava [8] observed that HDPE had a greater disagreement between theory

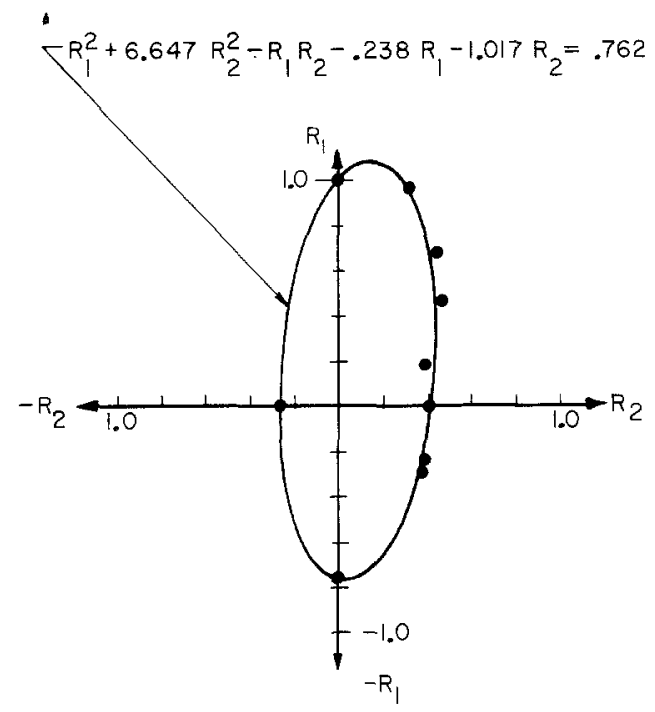

Figure 11 Comparison between theoretical yield locus based on the proposed criterion (Equation 9) and actual experimental results for oriented PP.

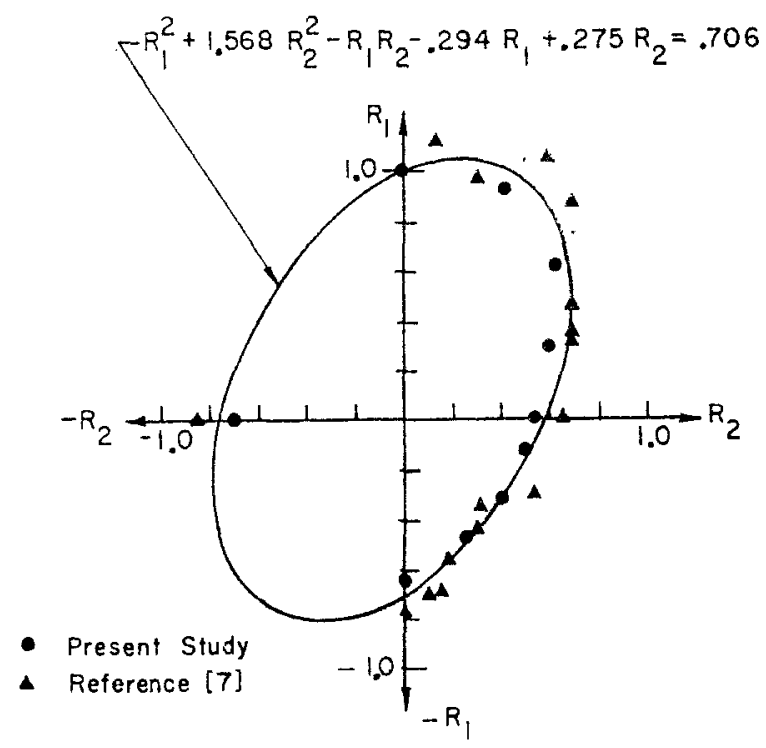

Figure 12 Comparison of predicted yield locus (Equation 9) with experimental results from two sources for oriented PC. and experiment and suggested it may be due to the higher relative creep rate of HDPE.

(3) The theoretical curve of PP is very much different from the corresponding curves for PC and HDPE, and the experimental data confirm this fact. This may be due to the formation of voids as evidenced by the significant drop in density when the PP was oriented.

(4) The increase in scatter of the test points at higher stress ratios (i.e. $R_{1} / R_{2}$ ) in the first quadrant seems to be prevalent. A possible cause is the relative error in pressure control. At high stress ratios, the pressure is relatively small, so small variations in pressure would cause a large percentage error. At low stress ratios, the pressure is relatively high and an equal error in pressure control would result in a much smaller percentage error. At present, a pressure control unit is being built which will reduce this type of error in future experiments.

(5) Buckling presented a problem in the uniaxial compression of unpressurized tubes for all three materials, and for $\mathrm{PP}$ it produced invalid results in the lower portion of the fourth quadrant of the yield locus. It is believed that this question of susceptibility to buckling in the oriented PP structure could be directly related to the formation of voids as mentioned earlier.

\section{Conclusion}

The yield locus studies of both amorphous and crystalline oriented polymers support the proposed yield criterion in its prediction of the macroscopic yielding of anisotropic, pressuredependent solids.

\section{Acknowledgements}

This work was conducted as part of a study under a National Science Foundation Grant DMR-7410355; that support is gratefully acknowledged. Mr William Durrant provided valuable assistance on some of the experimentation and most of the effort in producing the various figures. N. S. Sridharan assisted with most of the microscopic characterization studies, while Dr S. K. Bhateja of Dow Chemical most kindly provided the density gradient column and measured the densities of two of the materials.

\section{References}

1. N. BROWN, R. A. DUCKETT and I. M. WARD, Phil. Mag. 18 (1968) 483. 
2. J. G. RIDER and E. HARGREAVES, J..Polymer Sci. A-2 7 (1969) 829.

3. F. F. RAWSON and J. G. RIDER, ibid C 33 (1971) 87.

4. D. SHINOZAKI and G. W. GROVES, J, Mater, Sci. 8 (1973) 71.

5. R. HILL, "Plasticity" (Clarendon Press, Oxford, 1950) p. 317.

6. R. M. CADDELL, R. S. RAGHAVA and A. G.
ATKINS, J. Mater. Sci. 8 (1973) 1641.

7. R. S. RAGHAVA and R. M. CADDELL, Int. J. Mech. Sci. 16 (1974) 789.

8. R. S. RAGHAVA, Ph.D. Dissertation, The University of Michigan (December 1972).

9. R. S. RAGHAVA, R. M. CADDELL and G. S. Y. YEH, J. Mater. Sci. 8 (1973) 225.

Received 31 December 1976 and accepted 11 March 1977. 